\title{
Interleukin-18 predicts atherosclerosis progression in SIV-infected and uninfected rhesus monkeys (Macaca mulatta) on a high-fat/high-cholesterol diet
}

\author{
Jennifer H Yearley ${ }^{1}$, Dongling Xia ${ }^{1}$, Christine B Pearson ${ }^{1}$, Angela Carville ${ }^{1}$, Richard P Shannon ${ }^{2,3}$ \\ and Keith G Mansfield ${ }^{1, *}$
}

Interleukin (IL)-18 levels have been identified as important predictors of cardiovascular mortality and are often elevated in human immunodeficiency virus (HIV)-infected individuals. To investigate a possible function for IL-18 in atherogenesis in the context of early HIV infection, we used the simian immunodeficiency model of HIV infection. Acutely simian immunodeficiency virus-infected and uninfected rhesus monkeys (Macaca mulatta) on an atherogenic diet were evaluated prospectively for atherosclerotic lesion development relative to a panel of plasma markers including IL-18, IL-8, IL- $1 \beta$, IL-6, C-reactive protein, soluble vascular cell adhesion molecule-1, soluble E-selectin, and soluble intercellular adhesion molecule-1. Although no significant differences in lesion development were identified between groups after 35 days of infection, levels of plasma IL-18 measured 1 month before virus inoculation correlated significantly with atherosclerotic plaque cross-sectional area at the carotid bifurcation $(P<0.001, R=0.946)$, common iliac bifurcation $(P<0.01, R=0.789)$, and cranial abdominal aorta $(P<0.01, R=0.747)$, as well as with extent of CD3 + and CD68 + cellular infiltration in vascular lesions (both $P<0.001, R \geq 0.835$ ) in both groups. Atherosclerotic plaque area at the carotid and common iliac bifurcations also showed a weaker inverse correlation with baseline IL-8 levels, as did CD68 + signal area. Results implicate a strong role for IL-18 in early atherosclerosis progression and raise the possibility that the chronically elevated IL-18 levels seen in later stages of HIV infection may contribute significantly to accelerated atherogenesis in this population.

Laboratory Investigation (2009) 89, 657-667; doi:10.1038/labinvest.2009.29; published online 20 April 2009

KEYWORDS: atherosclerosis; endothelial activation markers; human immunodeficiency virus (HIV); interleukin-8 (IL-8); interleukin-18 (IL-18); simian immunodeficiency virus (SIV)

Atherosclerosis is a complex, lipid-dependent, arterial inflammatory disease. ${ }^{1,2}$ Interleukin-18 (IL-18) is a pleiotropic cytokine whose proatherogenic effects have been demonstrated in several murine models, ${ }^{3-6}$ and which has been shown to be expressed in human atherosclerotic plaques with increased expression in unstable plaques. ${ }^{7,8}$ Levels of circulating IL-18 have been positively correlated with carotid intima-media thickness and coronary plaque area in some studies, ${ }^{9,10}$ and multiple reports have identified IL-18 levels as important predictors of coronary events and cardiovascular mortality. ${ }^{11-15}$ IL-18 levels are commonly elevated in human immunodeficiency virus (HIV)-infected individuals, ${ }^{16-20}$ a population in which apparent accelerated atherogenesis has been reported since the early years of the epidemic. $^{21-23}$ Although the potential for a contributory role for HIV infection itself in accelerated atherosclerosis progression remains controversial, numerous studies suggest that HIV infection does directly or indirectly serve as an independent risk factor. ${ }^{24-29}$ HIV infection is intrinsically associated with dyslipidemias, endothelial dysfunction, and chronic immune activation, all of which have the potential to contribute to atherogenesis. ${ }^{30-34}$

Although multiple studies to date have examined atherosclerosis in chronically HIV-infected populations, evaluation of changes in the immediate postinfection period in HIVinfected people is generally not possible, as most individuals are not diagnosed until an indeterminate length of time after primary infection. Pathogenesis studies focused on early time

\footnotetext{
${ }^{1}$ Harvard Medical School, New England Primate Research Center, Southborough, MA, USA; ${ }^{2}$ Allegheny General Hospital, Pittsburgh, PA, USA and ${ }^{3}$ Department of Medicine, University of Pennsylvania School of Medicine, Philadelphia, PA, USA

${ }^{*}$ Correspondence: Dr KG Mansfield, DVM, Harvard Medical School, New England Primate Research Center, PO Box 9102, Southborough, MA 01772-9102, USA.

E-mail: keith_mansfield@hms.harvard.edu

Received 10 November 2008; revised 19 February 2009; accepted 9 March 2009
} 
points, ranging from infection to shortly after the establishment of viral set point, allow evaluation of changes resulting from initial high levels of viral replication and innate immune system activation associated with primary infection. ${ }^{35-37}$ In primate models of these earliest stages of HIV infection, circulating IL-18 levels show an initially transient increase, with level maxima corresponding to the time of peak viremia. ${ }^{37-39}$ This phenomenon could have long-term consequences in the context of chronic immune activation, and the later sustained increases in plasma IL-18 levels that characterize both HIV and simian immunodeficiency virus (SIV) infection even in the face of highly active antiretroviral therapy. ${ }^{16,18-20,39,40}$ In this report, the SIV model of HIV infection was used to investigate contributions of viral infection to atherogenesis in the early postinfection period. SIV and HIV share a close phylogenetic relationship, are characterized by tight structural and functional similarities, and produce similar disease states in susceptible hosts, culminating in profound immunodeficiency. ${ }^{41,42}$

In the current report, a group of 12 rhesus monkeys was maintained on an atherogenic diet for 6 months, after which half of the animals were infected with SIV. All animals were evaluated prospectively starting from 4 weeks before the time of SIV inoculation through killing, 9 weeks after inoculation. A panel of soluble plasma markers was monitored, including levels of the cytokines IL-18, IL-1 $\beta$, and IL-6; the chemokines IL-8 and monocyte chemoattractant protein-1 (MCP-1); the inflammatory biomarker C-reactive protein (CRP); and the endothelial activation markers soluble vascular cell adhesion molecule-1 (sVCAM-1), soluble E-selectin (sE-selectin), and soluble intercellular adhesion molecule-1 (sICAM-1). Levels of these markers, along with viral loads and CD4 T-cell counts, were evaluated for relationships to the size of atherosclerotic plaques at the carotid bifurcation, common iliac bifurcation, and cranial abdominal aorta at the time of necropsy, and for relationships to the extent of inflammatory cell infiltration, lipid accumulation, and cytokine, chemokine, and endothelial adhesion molecule expression within vascular sections.

\section{MATERIALS AND METHODS}

\section{Animals, Study Groups, Vascular Tissue Collection}

Twelve young adult male rhesus macaques (Macaca mulatta) were maintained on a commercially available atherogenic diet ad libitum (diet no. 57JI; LabDiet, St Louis, MO, USA; total fat $(39.35 \%$ of energy), saturated fat $(13.78 \%$ of energy), cholesterol ( $1 \%$ of diet on an as-fed basis), $n-6: n-3$ ratio of 7-10:1) for 6 months, after which six were intravenously inoculated with SIVmac239. All animals were killed 9 weeks later as previously described ${ }^{37}$ and were perfused with $4 \%$ paraformaldehyde in phosphate-buffered saline (PBS) at $95 \mathrm{~mm} \mathrm{Hg}$ (approximate mean arterial pressure) for $20 \mathrm{~min}$ before necropsy. All animals were housed at the New England Primate Research Center in accordance with standards of the Association for Assessment and Accreditation of Laboratory
Animal Care and Harvard Medical School's Animal Care and Use Committee.

For all animals, carotid arteries were collected bilaterally and evaluated histologically at the level of the bifurcation. The aorta was collected and evaluated histologically at the root, mid-thoracic region, level of the celiac artery, and common iliac bifurcation.

\section{Immunohistochemistry and Histochemical Staining}

Single-label immunohistochemistry was performed on formalin-fixed paraffin-embedded tissues following an $\mathrm{ABC}$ immunostaining technique as previously described. ${ }^{43}$ Formalin-fixed tissues were assessed using antibodies specific for CD3 (polyclonal; DakoCytomation, Carpinteria, CA, USA), CD68 (clone KP1; DakoCytomation), MCP-1 (polyclonal; R\&D Systems, Minneapolis, MN, USA), and SIV nef (clone KK75; donor Dr K Kent and Ms C Arnold, obtained from the NIBSC Centre for AIDS Reagents supported by EU Program EVA contract (QLKZ-CT-1999-00609) and the UK Medical Research Council). Sections were deparaffinized and rehydrated, followed by incubation in $3 \% \mathrm{H}_{2} \mathrm{O}_{2}$ in PBS, microwaving in sodium citrate buffer (Vector Laboratories, Burlingame, CA, USA), and sequential incubation with primary antibody, biotinylated secondary antibody, and horseradish peroxidase-conjugated avidin (ABC Standard or $\mathrm{ABC}$ Elite; Vector Laboratories). Antigen-antibody complex formation was detected by use of $3,3^{\prime}$-diaminobenzidine (DAB) chromogen (DakoCytomation).

Immunohistochemistry on cryosections was performed using antibody specific for VCAM-1 (clone 1G11.B1; Imgenex, San Diego, CA, USA), the ImmPRESS Immunolabeling kit (Vector Laboratories), and DAB chromogen (DakoCytomation).

Cryosections were stained for lipid using Oil Red O.

\section{Antigen Colocalization}

In antigen colocalization experiments, double-label immunohistochemistry was performed for pro-IL-18 (goat polyclonal; R\&D Systems) in combination with CD68 (clone KP1; DakoCytomation) and CD3 (polyclonal; DakoCytomation) as described for single-label immunohistochemistry, with the following exceptions. Incubation of pro-IL-18 antibody with secondary antibody was followed by incubation with ABC-alkaline phosphatase (Vectastain ABC-AP Standard Kit; Vector Laboratories) and detected with the Fast Red Substrate-Chromogen System (DakoCytomation). DAB chromogen (DakoCytomation) was used to detect antibodyantigen complex formation for CD68 and CD3 as in singlelabel immunohistochemistry. Labeled sections were imaged using an Olympus Vanox-S AHBS microscope interfaced with a liquid crystal tunable filter-based camera (CRI, Woburn, MA, USA) to allow spectral imaging for spatial resolution and colocalization of chromogens. Images were acquired from 420 to $720 \mathrm{~nm}$ at $20 \mathrm{~nm}$ steps and spectral components unmixed as previously described. ${ }^{44,45}$ In brief, 
the spectral absorbance of each chromogen was established from single stain controls and the contribution of each chromogen was calculated from this reference to determine the relative contribution at each pixel. Individual images were then pseudocolored and combined.

\section{Image Analysis}

Image analysis was performed with SigmaScan Pro 5.0 image analysis software (Systat Software Inc., Richmond, CA, USA). Atherosclerotic plaque cross-sectional areas were determined for the right carotid artery at the bifurcation, cranial abdominal aorta at the level of the celiac artery, and caudal abdominal aorta at the level of the common iliac bifurcation. Plaque cross-sectional areas were calculated by manual delimitation of plaque margins on images captured from $\mathrm{H} \& \mathrm{E}$ stained cross sections, and relativized to vessel cross-sectional areas, defined as extending from the luminal surface of the intima to the adventitial surface of the media.

Labeling for CD3, CD68, MCP-1, VCAM-1, and Oil Red O in vascular cross sections were quantitated as described below. CD3 + cells were quantitated by direct object counts alone and relativized to vessel cross-sectional area. CD68 and Oil Red O signal were quantitated by color thresholding relativized to vessel cross-sectional area. Endothelial MCP-1 signal was quantitated by imaging 100 consecutive endothelial cells starting from the region of highest endothelial MCP-1 signal on each slide and determining the percent positive cells based on captured images. VCAM-1 signal was quantitated by determining the cumulative length of VCAM1 signal-positive internal vascular circumference relativized to the total endothelium-lined internal vascular circumference. All image analysis was conducted blinded to group assignments.

\section{Lesion Scoring}

Vessels were scored on a 1-3 scale using American Heart Association lesion classification criteria. ${ }^{46,47}$ For purposes of statistical analysis, type I lesions were assigned a score of 1 , type II lesions a score of 2, type III lesions a score of 3, and type IIa lesions a score of 2.5. Vessels were evaluated blinded to group assignments.

\section{Enzyme-Linked Immunosorbent Assays and Luminex Analysis}

Commercial enzyme-linked immunosorbent assay kits for IL-18 (R\&D Systems), sVCAM-1 (R\&D Systems), monkey sE-selectin (Bender MedSystems, Burlingame, CA, USA), monkey sICAM-1 (Bender MedSystems), monkey CRP (Life Diagnostics, West Chester, PA, USA), and the MILLIPLEX ${ }^{\text {TM }}$ 23-plex nonhuman primate cytokine kit, including assays for IL-8, IL- $1 \beta$, and IL-6 (Millipore Corporation, Billerica, MA, USA) for Luminex analysis (Luminex Corporation, Austin, TX, USA), were run on batched samples of previously frozen plasma according to manufacturers' instructions. Time points analyzed included 4 weeks before SIV inoculation, 4 weeks after SIV inoculation, and immediately before killing.

\section{Virus and T Cells}

Plasma viral loads were determined by a TaqMan real-time reverse transcription polymerase chain reaction (RT-PCR) assay for SIV gag RNA as described in detail elsewhere. ${ }^{48,49} \mathrm{~T}$ cell subsets and total lymphocyte counts were monitored at regular intervals for all animals throughout the study period.

\section{Statistical Analysis}

Significance of differences between groups was determined using the $t$-test or Mann-Whitney rank-sum test as appropriate. Linear regression analysis was conducted to determine significance of correlations. All analyses were performed with commercially available software (SigmaStat 3.1; Systat Software). Probability values of $P<0.05$ were interpreted as significant.

\section{RESULTS \\ Atherosclerosis in Acutely SIV-Infected and Uninfected Rhesus Monkeys on an Atherogenic Diet}

Rhesus monkeys in both study groups rapidly developed atherosclerotic lesions. Lesions varied from scant subendothelial foam cell infiltrates to deeply stratified accumulations of macrophages and $\mathrm{T}$ cells with smooth muscle proliferation, individuated apoptotic cells, granular mineral deposition, and formation of early necrotic foci deep in the intima (Figure 1a). Determination of atherosclerotic plaque burdens for carotid artery, cranial abdominal aorta, and common iliac bifurcation identified a predominance of vessels without plaques, but a modest trend toward increased early plaque development among SIV-infected animals (Figure 1b). However, plaques markedly more extensive than the mean were identified in one uninfected animal and one SIV-infected animal (Figure 1b). Atherosclerotic plaques from both SIV-infected and uninfected animals contained low to moderate numbers of $\mathrm{T}$ cells, with T-cell involvement extending deeply into the media in the carotid of one SIVinfected animal (Figure 1c). Numbers of $\mathrm{T}$ cells in carotid plaques correlated strongly with plaque area $(P<0.001$, $R=0.890$; Figure 1d), and also with American Heart Association lesion classification $(P<0.001, R=0.628)$. Plaques from both SIV-infected and uninfected animals showed extensive macrophage involvement as evidenced by immunolabeling for CD68, with involvement extending focally into the media in the carotid lesion previously described (Figure 1e). As was seen for T cells, measures of CD68 + signal correlated strongly with carotid plaque cross-sectional area $(P<0.001, R=0.940$; Figure 1f), and with American Heart Association lesion-type classification $(P<0.001$, $R=0.705)$. 

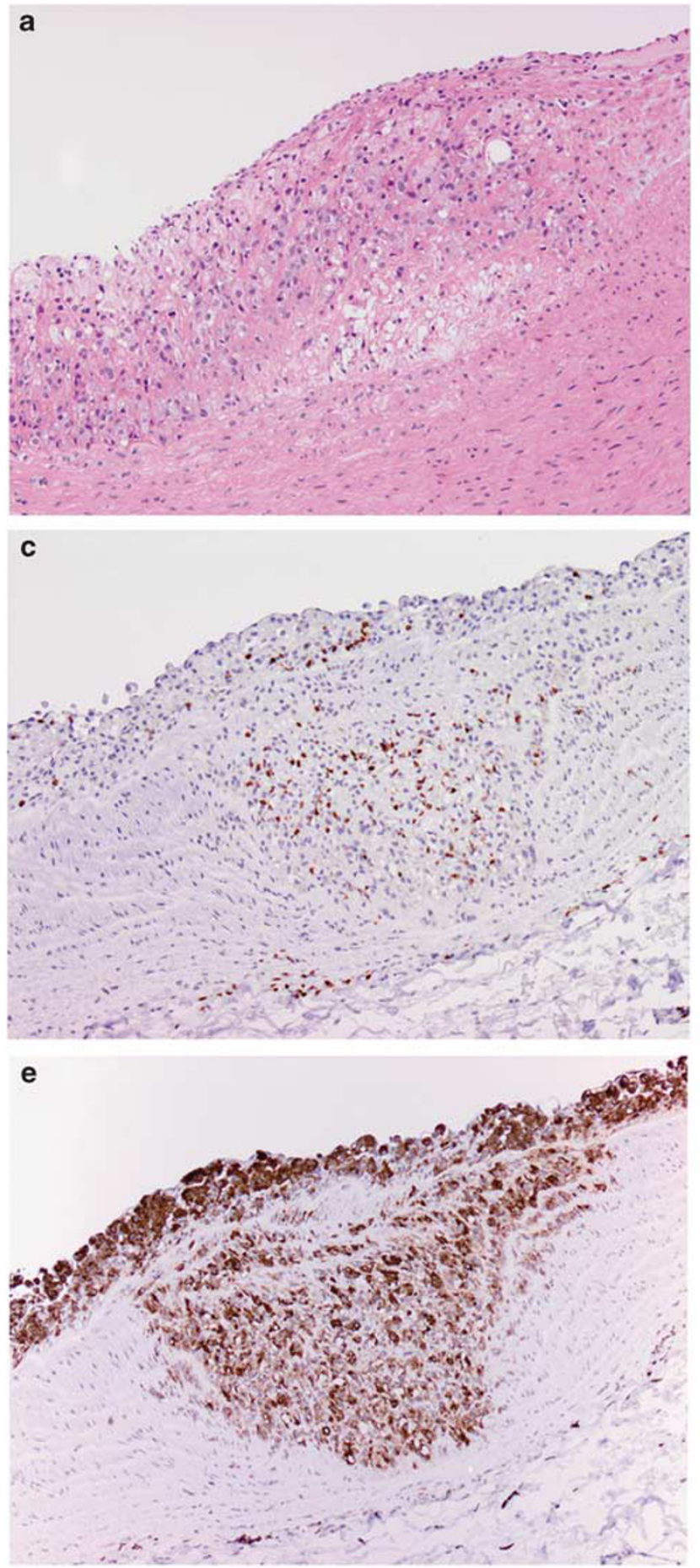
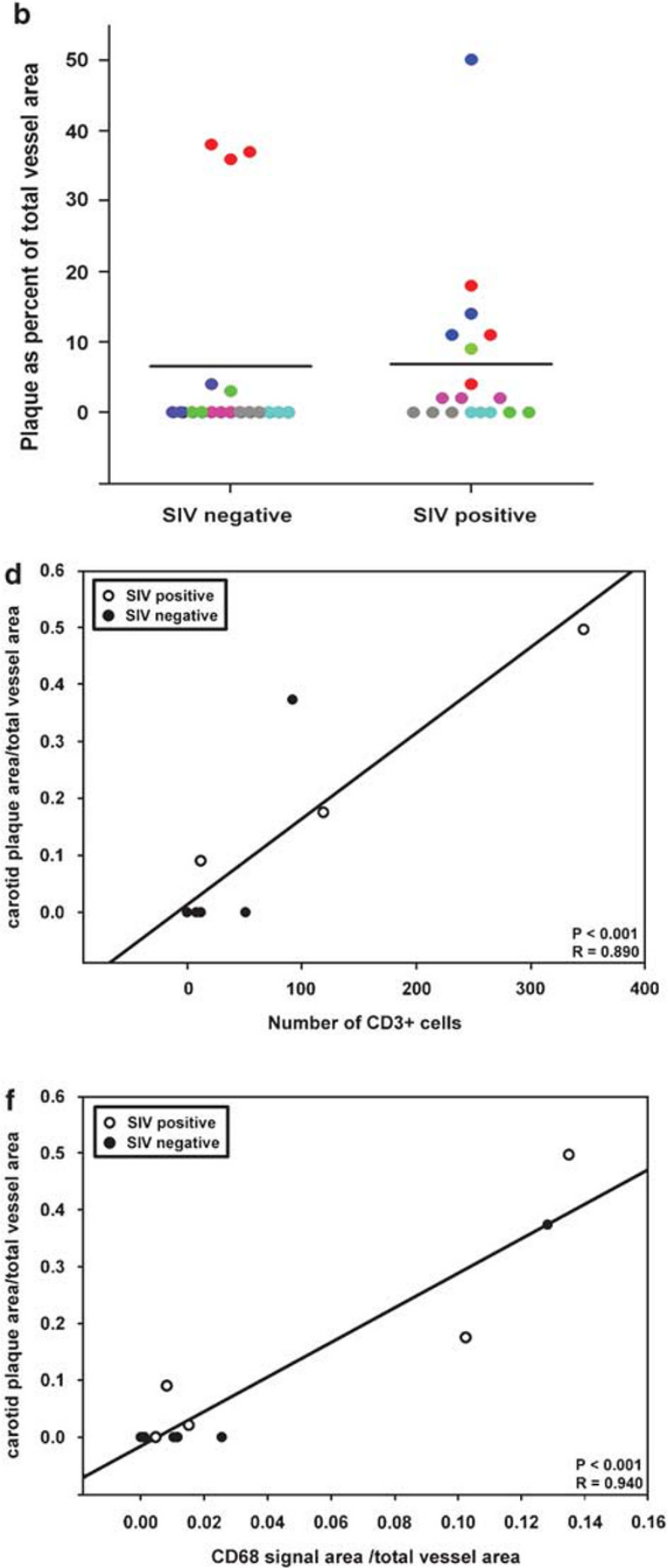

Figure 1 Atherosclerotic plaque character. (a) Thoracic aorta, SIV-negative animal. Stratified accumulations of intimal foam cells with smooth muscle proliferation, low numbers of lymphocytes, small pools of extracellular lipid, and an early deep focus of necrosis containing scant granular mineral (hematoxylin and eosin). (b) Summary of atherosclerotic plaque cross-sectional area findings at carotid bifurcation, cranial abdominal aorta, and common iliac bifurcation for each animal as a function of SIV infection status. Each of the three vessels from any single animal is represented by a single color. SIV-infected animals as a group showed a modest trend toward increased early plaque development, but one animal from each group demonstrated vessels with substantially greater plaque burdens than those of other animals in the cohort. (c) Carotid bifurcation, SIV-positive animal. CD3 immunolabeled cells are present in moderate numbers within the mild atherosclerotic lesion in the intima, but also extend deep into the media as part of an intense mixed inflammatory focus (CD3 immunohistochemistry with DAB chromogen and Mayer's hematoxylin counterstain). (d) Numbers of cells immunolabeling for CD3 within carotid plaques correlated strongly with plaque cross-sectional area $(P<0.001, R=0.890)$. (e) Carotid bifurcation, SIV-positive animal. CD68 immunolabeled cells constitute the primary population within the intimal lesion, but also extend deep into the media as part of an intense mixed inflammatory focus. (CD68 immunohistochemistry with DAB chromogen and Mayer's hematoxylin counterstain.) (f) CD68 signal area within carotid plaques correlated strongly with plaque cross-sectional area $(P<0.001, R=0.940)$. 


\section{Plasma IL-18 Predicts Early Lesion Progression in SIV- Infected and Uninfected Rhesus Monkeys}

Plasma IL-18 levels were measured in all animals 4 weeks before virus inoculation, 4 weeks after virus inoculation, and immediately before killing. At the 4 -week preinoculation time point, all animals had been on the atherogenic diet for 5 months, a time by which previous work has shown that peak IL-18 elevations associated with initiation of the atherogenic diet have resolved and stabilized to a new baseline. ${ }^{39}$ The 4week postinoculation time point corresponded to establishment of viral load set point after resolution of primary viremia, ${ }^{50,51}$ and the final time point allowed for evaluation of levels corresponding directly to the time at which tissues were harvested for lesion assessment and characterization.

IL-18 levels measured 4 weeks after SIV inoculation differed significantly between SIV-infected and uninfected groups $(P<0.02)$; however, no significant differences were present at other time points (Figure 2a). Baseline IL-18 levels, measured before virus inoculation, revealed two animals with levels significantly higher than for the remaining animals in the cohort $(P<0.05$; Figure $2 \mathrm{~b})$. At the 4 -week postinoculation time point, plasma IL-18 levels for these two animals remained essentially unchanged, whereas multiple other animals from the SIV-infected group showed infection-related increases (Figure 2c). By the time of killing 9 weeks after inoculation, plasma IL-18 levels had dropped uniformly for all animals, eliminating previous distinctions (Figure 2d).

Examining the relationship between levels of plasma IL-18 and measures of lesion progression, a tight set of correlations between baseline IL-18 levels and atherosclerotic lesion severity at necropsy was identified. Baseline plasma IL-18 showed close correlations with cross-sectional area of carotid plaques $(P<0.001, R=0.946$; Figure $2 \mathrm{e})$, plaques at the common iliac bifurcation $(P<0.01, R=0.789$; Figure $2 \mathrm{f})$, and at the cranial abdominal aorta $(P<0.01, R=0.747$; Figure $2 \mathrm{~g})$. Furthermore, baseline IL-18 levels correlated strongly with levels of T-cell infiltration $(P<0.001, R=0.835$; Figure $2 \mathrm{~h}), \mathrm{CD} 68+$ cell infiltration $(P<0.001, R=0.847$; Figure 2i), American Heart Association lesion-type classification $(P<0.05, R=0.604)$, and extent of vascular lipid accumulation $(P<0.02, R=0.687)$. No significant correlations were found between levels of plasma IL-18 at later time points and any parameter quantitating atherosclerotic lesion character or extent.

\section{Pro-IL-18 within Atherosclerotic Plaques is Expressed by Macrophages}

Within atherosclerotic plaques themselves, pro-IL-18 expression was extensive and strongly associated with macrophage foam cells, as demonstrated by colocalization of proIL-18 immunolabel with CD68 (Figure 3a and b). Although $\mathrm{T}$ cells and many other cell types are known to be capable of producing IL-18, ${ }^{6} \mathrm{~T}$ cells within the vascular lesions did not demonstrate expression of pro-IL-18 as indicated by absence of colocalization of pro-IL-18 immunolabel with CD3 in serial sections of the same vessels in which CD68 was evaluated.

\section{Baseline Plasma IL-8 Correlates Inversely with Lesion Progression in SIV-Infected and Uninfected Rhesus Monkeys}

Circulating levels of the chemokine IL- 8 were measured in animals of the acute infection cohort 4 weeks before virus inoculation, 4 weeks after virus inoculation, and immediately before killing. Plasma IL-8 levels did not differ significantly between infected and uninfected groups at any time point (Figure 4a). However, at baseline, two animals demonstrated plasma IL-8 levels substantially below the remainder of evaluated animals (Figure 4b). Baseline IL-8 levels correlated inversely with carotid plaque cross-sectional area $(P<0.04$, $R=-0.619$; Figure $4 \mathrm{c}$ ), as well as with plaque cross-sectional area at the common iliac bifurcation $(P<0.02, R=-0.685$; Figure $4 \mathrm{~d})$, and extent of CD68 + signal area in the most severely affected vessels $(P<0.01, R=-0.775$; Figure $4 \mathrm{e})$. No significant correlations were present between any measures of lesion severity and IL-8 levels at later time points.

\section{Plasma Viral Load, CD4 Count, and Endothelial Activation Marker Expression do not Correlate with Lesion Progression}

A moderate spectrum of plasma viral loads was evident at 4 weeks after inoculation, with differing patterns of viral load progression at the final time point (Figure 5a). However, viral loads did not correlate with any measure of vascular lesion character or extent. Furthermore, SIV-infected cells within atherosclerotic plaques were not identified in any vessel from this cohort of animals. Although CD4 T-cell counts were significantly lower in SIV-infected animals than controls at both 4 weeks after infection and killing $(P<0.05), \mathrm{CD} 4 \mathrm{~T}$-cell counts also did not correlate significantly with any measure of lesion character or extent.

Staining for lipid was extensive in all intimal plaques and in a subset of cases extended deep into the media (Figure 5b). Area of lipid signal relativized to total vessel cross-sectional area correlated positively with all measures of lesion severity including cross-sectional area of plaques in the carotid artery $(P<0.02, R=0.671)$, common iliac bifurcation $(P<0.03$, $R=0.637)$, and cranial abdominal aorta $(P<0.03$, $R=0.652)$, as well as American Heart Association lesion-type classification $(P<0.01, R=0.768)$, extent of T-cell infiltration $(P<0.03, R=0.647)$, extent of $\mathrm{CD} 68+$ signal area $(P<0.01, R=0.719)$, and baseline plasma IL-18, as previously noted $(P<0.02, R=0.687)$.

Levels of sVCAM-1, a systemic marker of endothelial activation, were significantly higher in infected animals than uninfected animals at 4 weeks after infection $(P<0.01$; Figure $5 c)$, remained higher at the time of killing $(P<0.05$; Figure $5 c)$, and correlated significantly with plasma viral loads $(P<0.02, R=0.691)$. However, levels of sVCAM-1 did not 
correlate significantly with any determined measure of lesion character or extent (data not shown).

Levels of sE-selectin, a second systemic marker of endothelial activation, did not differ significantly between groups at any time point (Figure 5d), and like sVCAM-1, did not correlate significantly with any determined measure of lesion character or extent.
Levels of sICAM-1, a further systemic marker of endothelial activation, also did not differ significantly between groups at any time point and, like sVCAM-1 and sE-selectin, did not correlate significantly with any measure of lesion character or extent (Figure 5e).

Circulating levels of the chemokine MCP-1 in plasma did not differ significantly between groups at any evaluated time
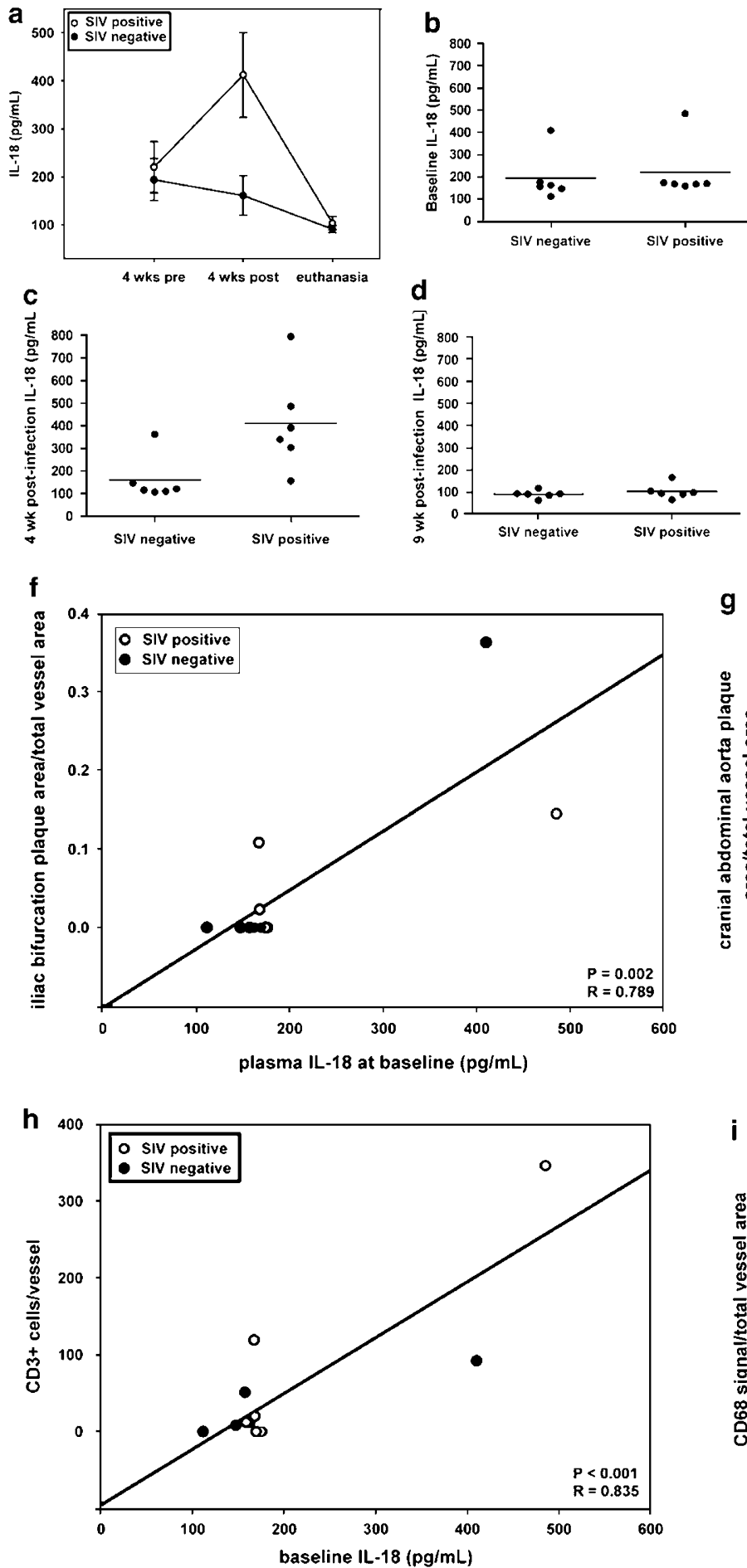
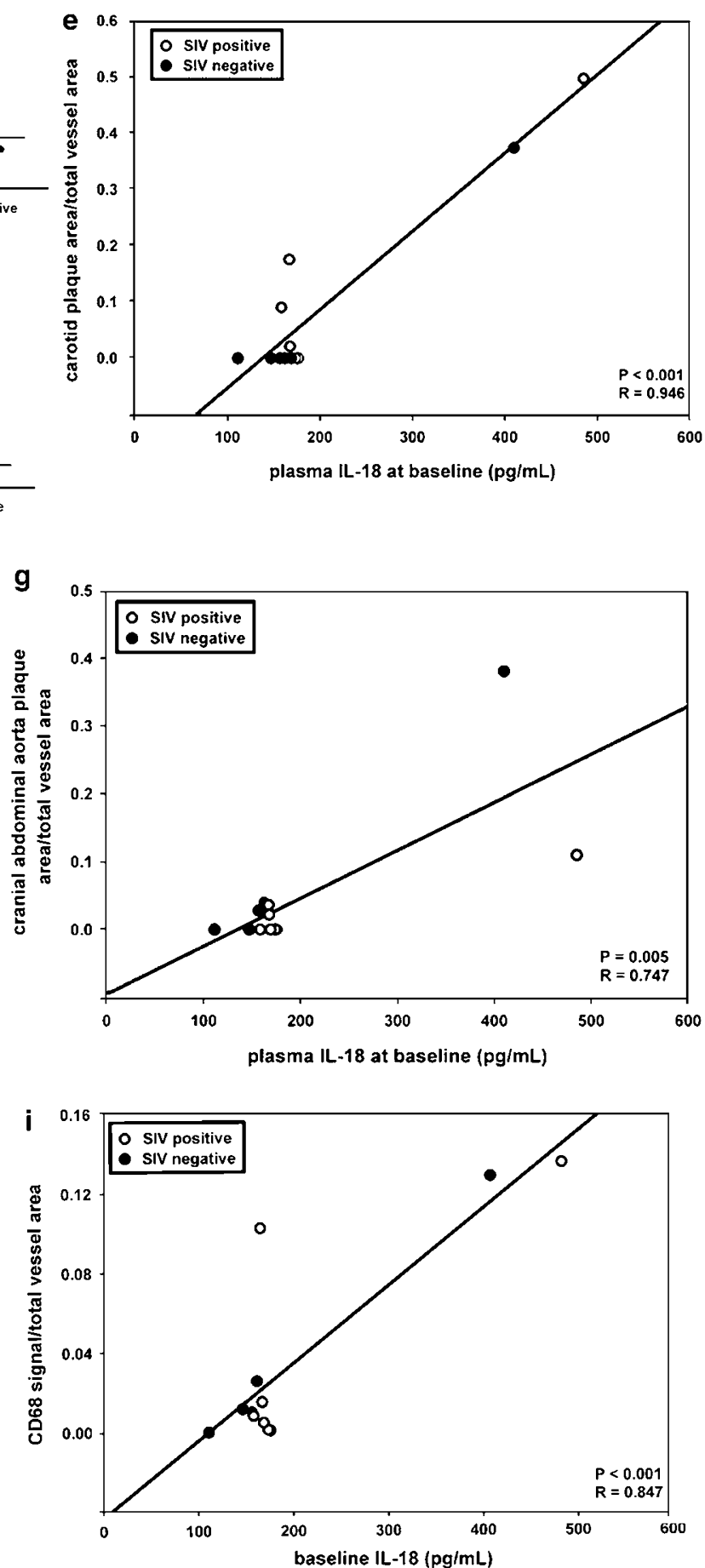

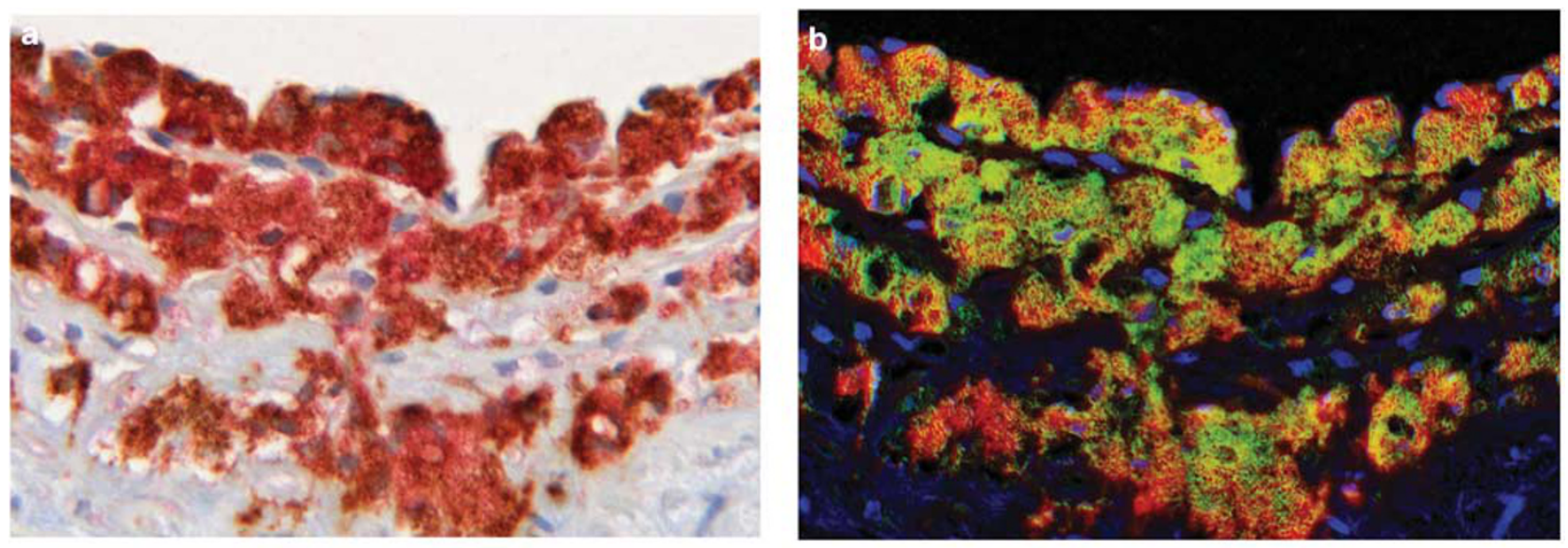

c

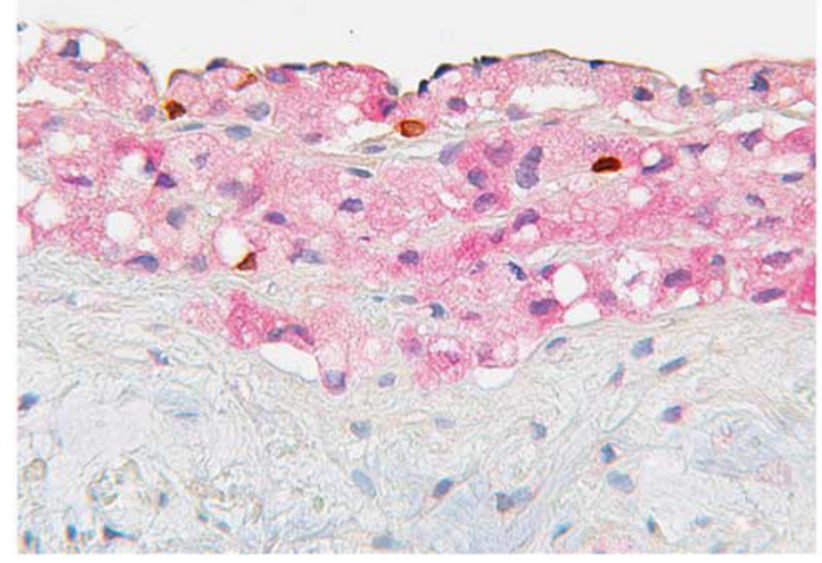

d

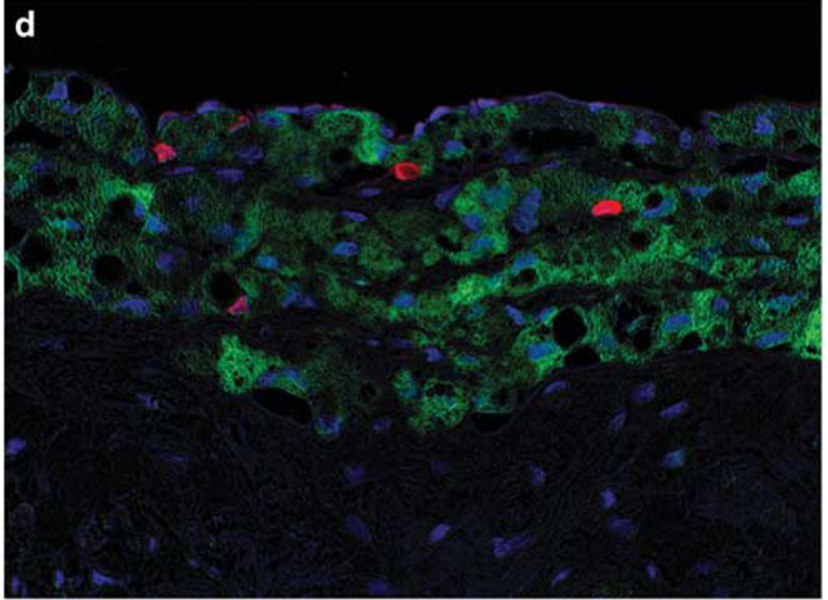

Figure 3 Serial sections of abdominal aorta demonstrating pro-IL-18 production in atherosclerotic lesions. (a) Immunolabeling for CD68 (brown) and pro-IL18 (pink) results in such extensive overlap in macrophage-rich regions of atherosclerotic plaques that very little differentiation of individuated signal is possible. (CD68 immunohistochemistry with DAB chromogen, pro-IL-18 immunohistochemistry with Fast Red chromogen, and Mayer's hematoxylin counterstain.) (b) Pseudofluorescent unmixed representation of the relative contributions of each chromogen in the stained tissue in panel (a), with pro-IL18 (green), CD68 (red), and colocalized signal (yellow). Pro-IL-18 signal is extensively colocalized with CD68, but regions of individuated CD68 signal beyond the areas of colocalization are present. (Spectral imaging of chromogens from immunohistochemistry in a.) (c) Immunolabeling for CD3 (brown) and pro-IL18 (pink) yields clearly differentiable signal for each (CD3 immunohistochemistry with DAB chromogen, pro-IL-18 immunohistochemistry with Fast Red chromogen, and Mayer's hematoxylin). (d) Pseudofluorescent unmixed representation of the relative contributions of each chromogen in the stained tissue in panel (a), with pro-IL-18 (green), and CD3 (red). Pro-IL-18 and CD3 labels show no colocalization (yellow) and occupy clearly distinct regions in the tissue. (Spectral imaging of chromogens from immunohistochemistry in (c).)

Figure 2 Plasma IL-18 levels and their correlations with measures of atherosclerotic lesion severity. (a) Plasma IL-18 levels were measured at three time points: 4 weeks before virus inoculation of the SIV-positive group, 4 weeks after virus inoculation of the SIV-positive group, and at the time of killing. IL-18 levels were significantly higher among SIV-infected animals than uninfected animals at the 4-week postinfection time point $(P<0.02)$. SIV positive, animals assigned to SIV-infected group; SIV negative, animals assigned to uninfected control group. (b) Plasma IL-18 levels measured at the 4-week preinoculation time point (baseline) were significantly higher for two animals than for other study group animals $(P<0.04)$. SIV positive, animals assigned to SIV-infected group; SIV negative, animals assigned to uninfected control group. (c) Plasma IL-18 levels measured 4 weeks after virus inoculation differed significantly between the SIV-infected and uninfected control groups $(P<0.02)$ due to infection-associated increases in circulating IL-18 among animals of the SIVinfected group. SIV positive, animals assigned to SIV-infected group; SIV negative, animals assigned to uninfected control group. (d) All significant distinctions in plasma IL-18 levels were lost at the 9-week postinfection time point, corresponding to the time of killing. SIV positive, animals assigned to SIV-infected group; SIV negative, animals assigned to uninfected control group. (e) Plasma IL-18 levels 4 weeks before virus inoculation (baseline) correlated significantly with carotid atherosclerotic plaque cross-sectional area at the time of necropsy $(P<0.001, R=0.946)$. (f) Plasma IL-18 levels 4 weeks before virus inoculation (baseline) correlated significantly with atherosclerotic plaque cross-sectional area at the common iliac bifurcation at the time of necropsy $(P<0.01, R=0.789)$. (g) Plasma IL-18 levels 4 weeks before virus inoculation (baseline) correlated significantly with atherosclerotic plaque cross-sectional area in the cranial abdominal aorta at the time of necropsy $(P<0.01, R=0.747)$. (h) Plasma IL-18 levels 4 weeks before virus inoculation (baseline) correlated significantly with extent of CD3 + cell infiltration in the most severely affected vessel per animal at the time of necropsy $(P<0.001, R=0.835)$. (i) Plasma IL18 levels 4 weeks before virus inoculation (baseline) correlated significantly with extent of CD68 + signal area in the most severely affected vessel per animal at the time of necropsy $(P<0.001, R=0.847)$. 

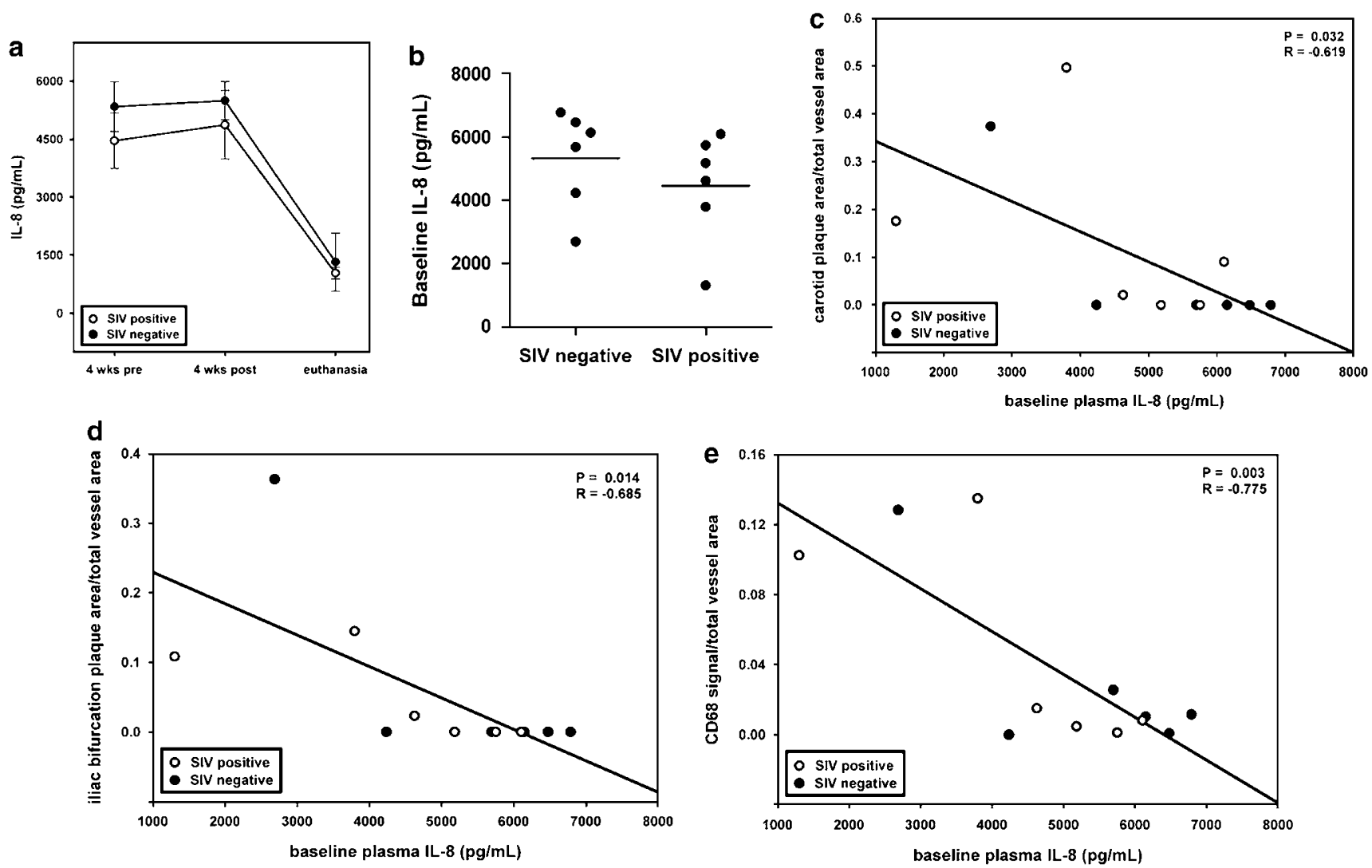

Figure 4 Plasma IL-8 levels and their correlations with measures of atherosclerotic lesion severity. (a) Levels of plasma IL-8 were measured 4 weeks before virus inoculation of the SIV-positive group, 4 weeks after virus inoculation of the SIV-positive group, and at the time of killing. Circulating IL-8 levels did not differ significantly between groups at any time point. SIV positive, animals assigned to SIV-infected group; SIV negative, animals assigned to uninfected control group. (b) A small subset of animals from both the SIV-negative and the SIV-positive groups demonstrated baseline IL-8 levels substantially lower than the mean. SIV positive, animals assigned to SIV-infected group; SIV negative, animals assigned to uninfected control group. (c) Atherosclerotic plaque cross-sectional area at the carotid bifurcation showed a significant inverse correlation with baseline plasma IL-8 levels, such that more extensive carotid plaques at necropsy were associated with lower baseline levels of IL-8 $(P<0.04, R=-0.619)$. (d) Atherosclerotic plaque cross-sectional area at the common iliac bifurcation showed a significant inverse correlation with baseline plasma IL-8 levels, such that more extensive plaques at necropsy were associated with lower baseline levels of IL-8 $(P<0.02, R=-0.685)$. (e) Extent of CD68 immunolabeling in the most severely affected vessel for each animal showed a significant inverse correlation with baseline plasma IL-8 levels, such that lower baseline plasma IL-8 levels were associated with more extensive CD68 + signal $(P<0.01, R=-0.775)$.

point and also did not correlate with any measure of lesion character or extent. MCP-1 expression in cells associated with vascular lesions was characteristically cytoplasmic and granular (Figure 5f). Quantification of percent MCP$1+$ endothelial cells revealed no significant differences between groups, but did demonstrate a positive correlation with American Heart Association lesion-type classifications $(P<0.03, R=0.455)$, and with levels of CRP at the 4-week preinoculation time point $(P=0.01, R=0.707)$. Neither circulating levels of CRP nor of IL-6 correlated with any examined measure of lesion character or extent, and levels of IL- $1 \beta$ were below the level of detection for all animals but one.

In a subset of vessels, endothelial VCAM-1 expression was strong, often with circumferentially discontinuous staining (Figure 5g). Quantification of VCAM-1 endothelial signal as a percent of total luminal circumference showed a trend to- ward greater levels of expression in SIV-negative animals (SIV negative: $23.3 \pm 4.1 \%$ vs SIV positive $9.8 \pm 5.4 \%$ (mean \pm standard error)), but no significant correlation with any measure of lesion character or extent.

\section{DISCUSSION}

In the current report, preinoculation plasma IL-18 levels, measured 5 months after initiation of atherogenic diet and slightly more than 3 months before killing, correlated significantly with all measured values of atherosclerotic lesion progression at necropsy, including plaque cross-sectional area at three anatomically distinct sites, extent of T-cell and macrophage infiltration, American Heart Association lesiontype classification, and extent of vascular lipid accumulation. These findings are in line with many reports in the recent literature implicating a function for IL-18 in atherogenesis. However, to the best of our knowledge, this is the first study 

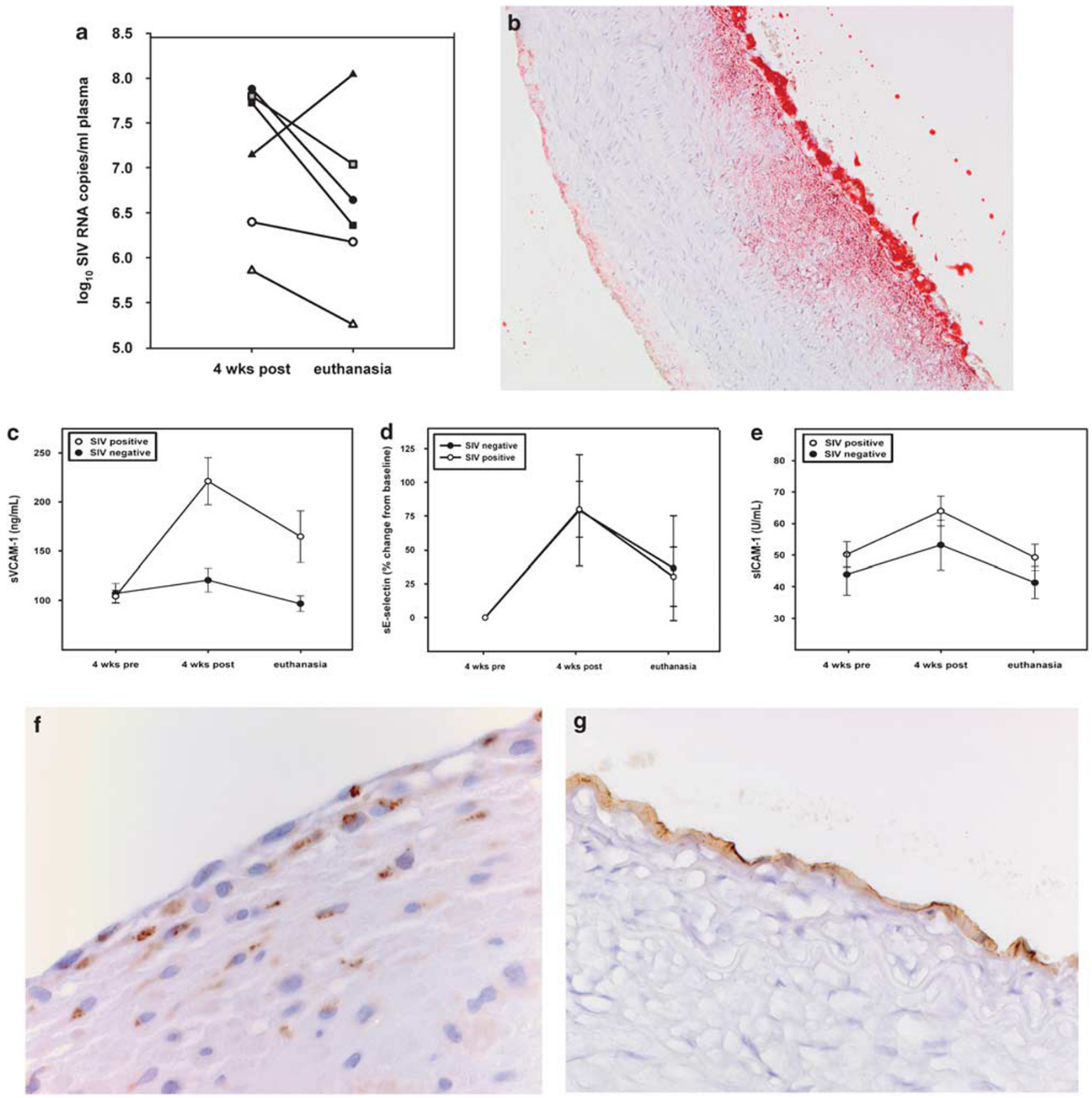

Figure 5 Viral load, vascular lipid accumulation, and markers of endothelial activation. (a) Plasma viral loads for SIV-infected animals at 4 weeks after infection (4 weeks post) and killing. (b) Cranial abdominal aorta, SIV-positive animal. There is intense positive staining for lipid in intimal foam cells along with focally extensive lipid droplet accumulation in medial smooth muscle (Oil Red O). (c) Soluble VCAM-1 (sVCAM-1) levels were significantly higher in SIVinfected animals than in uninfected animals at the 4-week postinfection time point $(P<0.01)$ and at the time of killing $(P<0.05) .4$ weeks pre, 4 weeks before virus inoculation of SIV-positive group; 4 weeks post, 4 weeks after virus inoculation of SIV-positive group; euthanasia, 9 weeks after virus inoculation of SIVpositive group. (d) The percent change in soluble E-selectin (sE-selectin) levels from baseline did not differ significantly between groups. 4 weeks pre, 4 weeks before virus inoculation of SIV-positive group; 4 weeks post, 4 weeks after virus inoculation of SIV-positive group; euthanasia, 9 weeks after virus inoculation of SIV-positive group. (e) Soluble ICAM-1 (sICAM-1) levels did not differ significantly between groups at any evaluated time point. 4 weeks pre, 4 weeks before virus inoculation of SIV-positive group; 4 weeks post, 4 weeks after virus inoculation of SIV-positive group; euthanasia, 9 weeks after virus inoculation of SIV-positive group. (f) Carotid bifurcation, SIV-positive animal. Granular cytoplasmic MCP-1 staining was present in a subset of endothelial cells in both SIV-positive and SIV-negative animals, as well as in scattered cells deeper within the intima. The percent of MCP-1 + endothelial cells correlated significantly with scores based on American Heart Association lesion-type classification $(P<0.03, R=0.455)$ and preinoculation levels of CRP $(P=0.01$, $R=0.707$ ) (MCP-1 immunohistochemistry with DAB chromogen and Mayer's hematoxylin counterstain). (g) Cranial abdominal aorta, SIV-negative animal. Many vessels showed strong, circumferentially discontinuous endothelial labeling for VCAM-1, but no significant correlations were identified between extent of endothelial VCAM- 1 expression and measures of atherosclerotic lesion character or extent (VCAM-1 immunohistochemistry with DAB chromogen and Harris hematoxylin counterstain). 
to demonstrate the predictive value of IL-18 so early in the process of atherogenesis, within a model where the time of first lesion initiation can be concretely defined, because rhesus monkeys do not develop atherosclerosis in the absence of atherogenic diet. Correlations between levels of IL-18 and measures of vascular lesion severity were lost at postinoculation week 4 , at which time significant elevations in IL18 were documented in the SIV-infected group. Although this result is initially surprising, it suggests that the transient virus-induced IL-18 elevations seen in primary infection are of substantially less importance than moderate persistent elevations in the steady state, a finding that may be of prognostic significance.

Baseline circulating IL-18 levels have been demonstrated to have prognostic value in multiple contexts. They have been identified as important predictors of coronary events and cardiovascular mortality, ${ }^{11-15}$ as predictors of accelerated disease progression in SIV infection, ${ }^{39}$ and as predictors of future systolic dysfunction in a rhesus model of HIV-associated cardiomyopathy. ${ }^{37}$ It is noteworthy that a number of polymorphisms within the proximal promoter region of the IL-18 gene in humans have been identified, with different haplotypes associated with different baseline levels of IL-18 expression and concomitantly differing cardiovascular risk. ${ }^{11,12,52-54}$ Given the substantial variation in baseline IL-18 levels in the current rhesus cohort, the possibility of similar polymorphisms in the rhesus population must also be entertained. Although the findings reported here do not support a function for early postinfection viral effects in promoting accelerated atherogenesis, they do raise the prospect that the elevated IL-18 levels seen chronically in HIVinfected individuals may be contributory to the accelerated atherosclerosis progression that has been documented in this population.

That baseline IL-8 levels were found to inversely correlate with both atherosclerotic plaque size and CD68 + cell involvement was surprising in that several studies have identified elevated levels of IL-8 as an independent risk factor for cardiovascular disease ${ }^{55-57}$ However, other work has shown that expression of the scavenger receptor gene SR-A in macrophages is inversely correlated with IL-8 gene expression. ${ }^{58}$ As SR-A is one of three scavenger receptors that together are responsible for uptake of greater than $90 \%$ of oxidized low-density lipoprotein, ${ }^{59,60}$ increasing scavenger receptor expression in association with decreasing IL-8 gene expression might provide a net increase in atherogenic effect, though exploring this possibility would require substantial further study.

To conclude, baseline IL-18 levels correlated strongly with atherosclerotic plaque area and inflammatory cell infiltration in this model, whereas endothelial activation markers, circulating systemic levels of MCP-1, IL- $1 \beta$, IL-6, CRP, plasma viral load, and CD4 counts showed no significant relationships with lesion size or character. Although viral infection did not appear significantly contributory to atherogenesis in the acute time frame, these findings raise the possibility that the chronically elevated levels of IL-18 seen in later stages of HIV infection may contribute significantly to accelerated atherogenesis in this population.

\section{ACKNOWLEDGEMENT}

This work was supported by National Institutes of Health grants RR00168, DK55510, HL075836, T32 RR007000, and K01 RR024120.

\section{DISCLOSURES}

The authors have no conflicts of interest to report.

1. Falk E. Pathogenesis of atherosclerosis. J Am Coll Cardiol 2006;47:C7C12.

2. Hansson GK, Libby $P$. The immune response in atherosclerosis: a double-edged sword. Nat Rev Immunol 2006;6:508-519.

3. Elhage $R$, Jawien J, Rudling $M$, et al. Reduced atherosclerosis in interleukin-18 deficient apolipoprotein E-knockout mice. Cardiovasc Res 2003;59:234-240.

4. Whitman SC, Ravisankar P, Daugherty A. Interleukin-18 enhances atherosclerosis in apolipoprotein $E(-/-)$ mice through release of interferon-gamma. Circ Res 2002;90:E34-E38.

5. Mallat Z, Corbaz A, Scoazec A, et al. Interleukin-18/interleukin-18 binding protein signaling modulates atherosclerotic lesion development and stability. Circ Res 2001;89:E41-E45.

6. Nakanishi K, Yoshimoto T, Tsutsui H, et al. Interleukin-18 regulates both Th1 and Th2 responses. Annu Rev Immunol 2001;19:423-474.

7. Gerdes N, Sukhova GK, Libby P, et al. Expression of interleukin (IL)-18 and functional IL-18 receptor on human vascular endothelial cells, smooth muscle cells, and macrophages: implications for atherogenesis. J Exp Med 2002;195:245-257.

8. Mallat Z, Corbaz A, Scoazec A, et al. Expression of interleukin-18 in human atherosclerotic plaques and relation to plaque instability. Circulation 2001;104:1598-1603.

9. Yamagami H, Kitagawa K, Hoshi T, et al. Associations of serum IL-18 levels with carotid intima-media thickness. Arterioscler Thromb Vasc Biol 2005;25:1458-1462.

10. Hulthe J, McPheat W, Samnegard A, et al. Plasma interleukin (IL)-18 concentrations is elevated in patients with previous myocardial infarction and related to severity of coronary atherosclerosis independently of C-reactive protein and IL-6. Atherosclerosis 2006;188:450-454.

11. Thompson SR, Novick D, Stock CJ, et al. Free interleukin (IL)-18 levels, and the impact of IL18 and IL18BP genetic variation, in CHD patients and healthy men. Arterioscler Thromb Vasc Biol 2007;27:2743-2749.

12. Tiret L, Godefroy $T$, Lubos $E$, et al. Genetic analysis of the interleukin-18 system highlights the role of the interleukin-18 gene in cardiovascular disease. Circulation 2005;112:643-650.

13. Blankenberg S, Tiret L, Bickel C, et al. Interleukin-18 is a strong predictor of cardiovascular death in stable and unstable angina. Circulation 2002;106:24-30.

14. Espinola-Klein C, Rupprecht HJ, Bickel C, et al. Inflammation, atherosclerotic burden and cardiovascular prognosis. Atherosclerosis 2007;195:e126-e134.

15. Blankenberg S, Luc G, Ducimetiere $P$, et al. Interleukin-18 and the risk of coronary heart disease in European men: the Prospective Epidemiological Study of Myocardial Infarction (PRIME). Circulation 2003;108:2453-2459.

16. Song W, Wilson CM, Allen S, et al. Interleukin 18 and human immunodeficiency virus type I infection in adolescents and adults. Clin Exp Immunol 2006;144:117-124.

17. Lindegaard $B$, Hansen $A B$, Gerstoft J, et al. High plasma level of interleukin-18 in HIV-infected subjects with lipodystrophy. J Acquir Immune Defic Syndr 2004;36:588-593.

18. Ahmad R, Sindhu ST, Toma E, et al. Elevated levels of circulating interleukin-18 in human immunodeficiency virus-infected individuals: role of peripheral blood mononuclear cells and implications for AIDS pathogenesis. J Virol 2002;76:12448-12456.

19. Stylianou E, Bjerkeli V, Yndestad A, et al. Raised serum levels of interleukin-18 is associated with disease progression and may 
contribute to virological treatment failure in HIV-1-infected patients. Clin Exp Immunol 2003;132:462-466.

20. Wiercinska-Drapalo A, Jaroszewicz J, Flisiak R, et al. Plasma interleukin18 is associated with viral load and disease progression in HIV-1infected patients. Microbes Infect 2004;6:1273-1277.

21. Tabib A, Greenland T, Mercier I, et al. Coronary lesions in young HIVpositive subjects at necropsy. Lancet 1992;340:730.

22. Paton $\mathrm{P}$, Tabib A, Loire $\mathrm{R}$, et al. Coronary artery lesions and human immunodeficiency virus infection. Res Virol 1993;144:225-231.

23. Tabib A, Leroux C, Mornex JF, et al. Accelerated coronary atherosclerosis and arteriosclerosis in young humanimmunodeficiency-virus-positive patients. Coron Artery Dis 2000;11:41-46.

24. Lorenz MW, Stephan C, Harmjanz A, et al. Both long-term HIV infection and highly active antiretroviral therapy are independent risk factors for early carotid atherosclerosis. Atherosclerosis 2008;196:720-726.

25. Hsue PY, Lo JC, Franklin A, et al. Progression of atherosclerosis as assessed by carotid intima-media thickness in patients with HIV infection. Circulation 2004;109:1603-1608.

26. Mangili A, Gerrior J, Tang AM, et al. Risk of cardiovascular disease in a cohort of HIV-infected adults: a study using carotid intima-media thickness and coronary artery calcium score. Clin Infect Dis 2006;43:1482-1489.

27. Klein D, Hurley LB, Quesenberry Jr CP, et al. Do protease inhibitors increase the risk for coronary heart disease in patients with HIV-1 infection? J Acquir Immune Defic Syndr 2002;30:471-477.

28. van Wijk JP, de Koning EJ, Cabezas MC, et al. Functional and structural markers of atherosclerosis in human immunodeficiency virus-infected patients. J Am Coll Cardiol 2006;47:1117-1123.

29. Periard D, Cavassini M, Taffe $P$, et al. High prevalence of peripheral arterial disease in HIV-infected persons. Clin Infect Dis 2008;46: 761-767.

30. Passalaris JD, Sepkowitz KA, Glesby MJ. Coronary artery disease and human immunodeficiency virus infection. Clin Infect Dis 2000;31: 787-797.

31. Fisher SD, Miller TL, Lipshultz SE. Impact of HIV and highly active antiretroviral therapy on leukocyte adhesion molecules, arterial inflammation, dyslipidemia, and atherosclerosis. Atherosclerosis 2006;185:1-11.

32. Kline ER, Sutliff RL. The roles of HIV-1 proteins and antiretroviral drug therapy in HIV-1-associated endothelial dysfunction. J Investig Med 2008;56:752-769.

33. Grunfeld C, Pang M, Doerrler W, et al. Lipids, lipoproteins, triglyceride clearance, and cytokines in human immunodeficiency virus infection and the acquired immunodeficiency syndrome. J Clin Endocrinol Metab 1992;74:1045-1052.

34. Feingold KR, Krauss RM, Pang $M$, et al. The hypertriglyceridemia of acquired immunodeficiency syndrome is associated with an increased prevalence of low density lipoprotein subclass pattern B. J Clin Endocrinol Metab 1993;76:1423-1427.

35. Mattapallil JJ, Douek DC, Hill B, et al. Massive infection and loss of memory CD4+ T cells in multiple tissues during acute SIV infection. Nature 2005;434:1093-1097.

36. Sasseville VG, Lane JH, Walsh D, et al. VCAM-1 expression and leukocyte trafficking to the CNS occur early in infection with pathogenic isolates of SIV. J Med Primatol 1995;24:123-131.

37. Yearley JH, Mansfield KG, Carville AA, et al. Antigenic stimulation in the simian model of HIV infection yields dilated cardiomyopathy through effects of TNFalpha. AIDS 2008;22:585-594.

38. Kaizu M, Ami Y, Nakasone T, et al. Higher levels of IL-18 circulate during primary infection of monkeys with a pathogenic SHIV than with a nonpathogenic SHIV. Virology 2003;313:8-12.

39. Mansfield KG, Carville A, Wachtman L, et al. A diet high in saturated fat and cholesterol accelerates simian immunodeficiency virus disease progression. J Infect Dis 2007;196:1202-1210.
40. Torre D, Speranza F, Martegani R, et al. Circulating levels of IL-18 in adult and paediatric patients with HIV-1 infection. Aids 2000;14: 2211-2212.

41. Gao F, Bailes E, Robertson DL, et al. Origin of HIV-1 in the chimpanzee Pan troglodytes troglodytes. Nature 1999;397:436-441.

42. Desrosiers RC. The simian immunodeficiency viruses. Annu Rev Immunol 1990;8:557-578.

43. Yearley JH, Pearson C, Shannon RP, et al. Phenotypic variation in myocardial macrophage populations suggests a role for macrophage activation in SIV-associated cardiac disease. AIDS Res Hum Retroviruses 2007;23:515-524.

44. Levenson RM, Mansfield JR. Multispectral imaging in biology and medicine: slices of life. Cytometry A 2006;69:748-758.

45. Mansfield JR, Hoyt C, Levenson RM. Visualization of microscopy-based spectral imaging data from multi-label tissue sections. Curr Protoc Mol Biol 2008;84:14.9.1-14.19.15.

46. Stary $\mathrm{HC}$, Chandler AB, Dinsmore RE, et al. A definition of advanced types of atherosclerotic lesions and a histological classification of atherosclerosis. A report from the Committee on Vascular Lesions of the Council on Arteriosclerosis, American Heart Association. Circulation 1995;92:1355-1374.

47. Stary $H C$, Chandler $A B$, Glagov $S$, et al. A definition of initial, fatty streak, and intermediate lesions of atherosclerosis. A report from the Committee on Vascular Lesions of the Council on Arteriosclerosis, American Heart Association. Circulation 1994;89:2462-2478.

48. Suryanarayana K, Wiltrout TA, Vasquez GM, et al. Plasma SIV RNA viral load determination by real-time quantification of product generation in reverse transcriptase-polymerase chain reaction. AIDS Res Hum Retroviruses 1998;14:183-189.

49. Lifson JD, Rossio JL, Piatak Jr M, et al. Role of CD8(+) lymphocytes in control of simian immunodeficiency virus infection and resistance to rechallenge after transient early antiretroviral treatment. J Virol 2001;75:10187-10199.

50. Staprans SI, Dailey PJ, Rosenthal A, et al. Simian immunodeficiency virus disease course is predicted by the extent of virus replication during primary infection. J Virol 1999;73:4829-4839.

51. Lifson JD, Nowak MA, Goldstein $\mathrm{S}$, et al. The extent of early viral replication is a critical determinant of the natural history of simian immunodeficiency virus infection. J Virol 1997;71:9508-9514.

52. Thompson SR, Humphries SE. Interleukin-18 genetics and inflammatory disease susceptibility. Genes Immun 2007;8:91-99.

53. Thompson SR, McCaskie PA, Beilby JP, et al. IL18 haplotypes are associated with serum IL-18 concentrations in a population-based study and a cohort of individuals with premature coronary heart disease. Clin Chem 2007;53:2078-2085.

54. Barbaux S, Poirier O, Godefroy $\mathrm{T}$, et al. Differential haplotypic expression of the interleukin-18 gene. Eur J Hum Genet 2007;15: 856-863.

55. Inoue $T$, Komoda $H$, Nonaka $M$, et al. Interleukin-8 as an independent predictor of long-term clinical outcome in patients with coronary artery disease. Int J Cardiol 2008;124:319-325.

56. Boekholdt SM, Peters RJ, Hack CE, et al. IL-8 plasma concentrations and the risk of future coronary artery disease in apparently healthy men and women: the EPIC-Norfolk prospective population study. Arterioscler Thromb Vasc Biol 2004;24:1503-1508.

57. Braunersreuther $\mathrm{V}$, Mach $\mathrm{F}$, Steffens $\mathrm{S}$. The specific role of chemokines in atherosclerosis. Thromb Haemost 2007:97:714-721.

58. Martin-Fuentes $P$, Civeira F, Recalde $D$, et al. Individual variation of scavenger receptor expression in human macrophages with oxidized low-density lipoprotein is associated with a differential inflammatory response. J Immunol 2007;179:3242-3248.

59. Steinberg D. Atherogenesis in perspective: hypercholesterolemia and inflammation as partners in crime. Nat Med 2002;8:1211-1217.

60. Miller $\mathrm{Yl}$, Chang MK, Binder $\mathrm{CJ}$, et al. Oxidized low density lipoprotein and innate immune receptors. Curr Opin Lipidol 2003;14:437-445. 